\title{
Physical legacy of freshwater bivalves: Effects of habitat complexity on the taxonomical and functional diversity of invertebrates
}

\author{
Martina I. Ilarri a,* Luís Amorim ${ }^{\text {b }}$, Allan T. Souza ${ }^{c}$, Ronaldo Sousa ${ }^{a, b}$

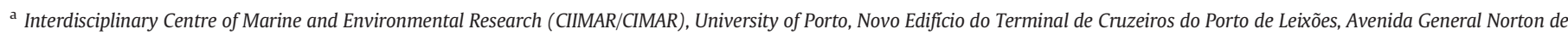 \\ Matos, s/n, 4450-208 Matosinhos, Portugal \\ b CBMA - Centre of Molecular and Environmental Biology, Department of Biology, University of Minho, Campus de Gualtar, 4710-057 Braga, Portugal \\ ${ }^{\mathrm{c}}$ Institute of Hydrobiology, Biology Centre of the Czech Academy of Sciences, Na Sádkách 7, 37005 České Budějovice, Czech Republic
}

\section{H I G H L I G H T S}

- Dominant invasive bivalves can contribute to habitat homogeneity.

- Reduction of bivalve identities changed the macroinvertebrates density.

- Functional diversity of the associated assemblages was not influenced.

- Bivalve identity is relevant to the density and function of associated assemblages.

\section{G R A P H I C A L A B S T R A C T}

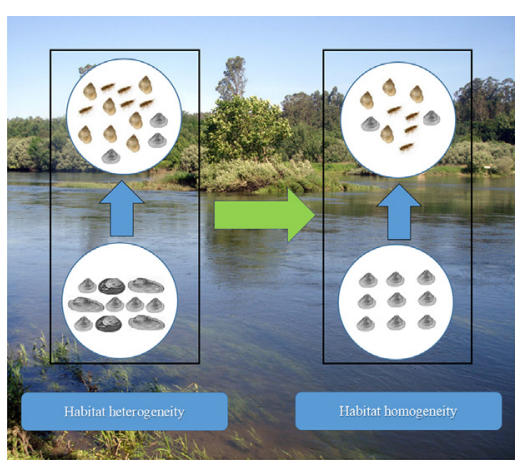

\section{A B S T R A C T}

Bivalves may play a major role in structuring aquatic communities. This may be especially relevant in aquatic communities dominated by non-native invasive bivalves, which can contribute to the increase of habitat homogenization. In this study, we assess how habitat homogenization, through the reduction of empty bivalve shells identities, influences the macroinvertebrate assemblages. Towards this end, a manipulative experiment with the empty shells of two native (Potomida littoralis and Unio delphinus) and one non-native (Corbicula fluminea) species was performed. Seven treatments were prepared, three of them consisting of homogeneous substrates using shells of one species, and four of them consisting in heterogeneous substrates using more than one species. The associated fauna colonizing different treatments was analyzed through taxonomic and trait-based approaches. Our results showed that the substrate complexity influenced the density of macroinvertebrates, with the heterogeneous treatments significantly yielding more dense assemblages. Also, the trait patterns differed among the levels of habitat heterogeneity, influencing mainly organisms that feed on microphytes of both small and big sizes, that inhabit areas with slow to moderate water flow, and that have short and long live cycles. Further, the functional diversity was not influenced by the substrate heterogeneity. Therefore, the habitat homogenization, through the accumulation of non-native $C$. fluminea empty shells in the river bottom, did not affect the functional diversity of the macroinvertebrate assemblages.

(c) 2018 Elsevier B.V. All rights reserved.

\footnotetext{
* Corresponding author.

E-mail address: martinailarri@gmail.com. (M.I. Ilarri).
} 


\section{Introduction}

Bivalves are important ecosystem engineers playing an important role in structuring aquatic communities (Gutiérrez et al., 2003; Sousa et al., 2009; Zaiko et al., 2009; Linares et al., 2017). They can alter the physical structure of the benthic environment through their shells in creating colonizable biogenic substrate for several species (Gutiérrez et al., 2003; Bódis et al., 2014; Burdon et al., 2014). The substratum provided by the presence of bivalve shells are used by the associated fauna to avoid predators and competitors (Ilarri et al., 2012, 2014, 2015a), as well as to reduce the physical and/or physiological stress as they can be responsible for changes in the current velocity (Gutiérrez et al., 2003; Erwin, 2008).

In recent years, the frequency and intensity of occurrence of bivalve massive mortality events have increased (Ilarri et al., 2011; Sousa et al., 2012; Bódis et al., 2014; Leuven et al., 2014). These unusual environmental conditions can trigger modifications in the dynamics of bivalve populations leading, in severe cases, to massive mortalities, and hence, the deposition of their shells into the river bed or adjacent river banks (Ilarri et al., 2015b; Novais et al., 2015b, 2017). After massive die-off of bivalves, the species composition and dynamic of the benthic compartment can be severely altered by changing the species pool of a given area often leading to homogenization due to the loss of rare species and the dominance of opportunistic species (Haag and Warren, 2008). The effects of bivalve die-offs can be more severe to the non-native than to native bivalve species (e.g. Sousa et al., 2007, 2008c; Haag and Warren, 2008). However, in most cases, non-native invasive bivalves have rapidly recovered from the mortality events, quickly returning to their previous densities, unlike native species (Sousa et al., 2008a).

In general, abundant and dominant invasive bivalves contribute to the deposit of large quantities of shells of the same species (i.e. habitat homogenization). Ecologically, habitat homogenization can imply changes of the physical structure of the habitat. For example, changes in habitat heterogeneity have been accompanied in the River Minho since the introduction of the Asian clam Corbicula fluminea in 1989 (Araujo et al., 1993). C. fluminea invasion has contributed to drastical declines in the density of native bivalve species (i.e. Anodonta anatina (Linnaeus, 1785), Potomida littoralis (Cuvier, 1798) and Unio delphinus (Spengler, 1793)) in the River Minho (Sousa et al., 2008c). Now, this invasive species is responsible for the majority (99\%) of empty shells deposited in the river bottom and adjacent river banks of the last $70 \mathrm{~km}$ of the river, with some areas reaching empty shell densities of $>2000$ ind $\cdot \mathrm{m}^{-2}$ (Ilarri et al., 2015b). Given these numbers, it is fundamental to evaluate the influence that the changes in habitat heterogeneity may have on the macroinvertebrate associated fauna.

For this, a manipulative experiment was performed and the resulting biotic data analyzed using both taxonomic and trait-based approaches. The former has been widely used in several community studies over the last decades. Whilst the latter is gaining more attention recently, given that it disentangles valuable ecological information often hidden in the classical taxonomical approach (Weigel et al., 2016). Despite of its relevance, the trait approach is still rarely applied in studies using macroinvertebrates and even rarer are studies that incorporate both taxonomical and trait-based approaches (but see Van der Linden et al., 2012, 2016; Clare et al., 2015; Wong and Dowd, 2015). To this end, the present study aimed at understanding the role played by substrate heterogeneity (using different shell identities) on the structure of macroinvertebrate assemblages using the traditional taxonomy and the trait-based approaches. The null hypothesis of the present study is that the substrate heterogeneity created by different shell identities has no effect on the macroinvertebrate diversity (both taxonomic and functional). We predict that treatments with higher heterogeneity will have a higher taxonomic and functional diversity in the associated macroinvertebrate assemblages.

\section{Material and methods}

\subsection{Study area}

The study was performed in the River Minho (NW of the Iberian Peninsula). This river drains a hydrological basin with a total area of $17,080 \mathrm{~km}^{2}$, of which $95 \%$ is located in Spain and $5 \%$ in Portugal. The River Minho originates in Serra da Meira, in the province of Lugo, Spain, and has an extent of approximately $300 \mathrm{~km}$. The last $70 \mathrm{~km}$ are located in the Portuguese/Spanish border, draining NNE-SSW into the Atlantic Ocean (Costa-Dias et al., 2010). The study was conducted in the village of Cortes, Monção (42 $\left.04^{\prime} 36.90^{\prime \prime} \mathrm{N} ; 8^{\circ} 30^{\prime} 54.42^{\prime \prime} \mathrm{W}\right)$ (Fig. 1). The site is located in a very shallow area (in summer reaches $1 \mathrm{~m}$ deep) with permanent freshwater conditions. This site is colonized mainly by the faucet snail Bithynia tentaculata, worms of the subclass Oligochaeta and C. fluminea (with $>2000$ ind $\cdot \mathrm{m}^{-2}$ ) (Ilarri et al., 2015b). Native bivalves such as A. anatina, P. littoralis, U. delphinus and several species from the Psidium genus also occur in the site (Sousa et al., 2005; Ilarri et al., 2015a). Its substratum composition consists mainly of pebbles, cobbles, coarse and medium sand, macrophytes and $C$. fluminea shells (live and empty). The water current in the site vary seasonally from moderate (summer and autumn) to strong (winter and spring).

\subsection{Experimental design and laboratory procedures}

To evaluate the colonization of macroinvertebrates through different substrates composition we considered select the most representative native and non-native bivalve species of the study site capable to increase the substrate area for the macroinvertebrates colonization. Empty shells of three species of bivalves, two native (P. littoralis and $U$. delphinus) and one non-native ( $C$. fluminea) were used. The three species live in sympatry in the study area but with a great dominance of $C$. fluminea, being $U$. delphinus and $P$. littoralis present in low densities (Sousa et al., 2005, 2007). Seven treatments were prepared, three of them consisting in homogeneous substrates, in which only shells of one species, hereafter Cor ( $C$. fluminea), Pot ( $P$. littoralis) and Uni (U. delphinus), and four of them in heterogeneous substrates, in which shells of more than one species were used, hereafter CorPot (C. fluminea and P. littoralis), CorUni (C. fluminea and U. delphinus), PotUni ( $P$. littoralis and U. delphinus) and CorPotUni (C. fluminea, $P$. littoralis and $U$. delphinus).

The treatments were standardized and for this an identical sum of shell's outer area $\left(1000 \mathrm{~cm}^{2}\right)$ was considered (i.e. homogeneous: $1000 \mathrm{~cm}^{2}$, heterogeneous with two species: $500 \mathrm{~cm}^{2}+500 \mathrm{~cm}^{2}$, and heterogeneous with three species: $333.3 \mathrm{~cm}^{2}+333.3 \mathrm{~cm}^{2}+333.3 \mathrm{~cm}^{2}$ ). To avoid bias due to heterogeneities in size we used shells close to the average size of each species present in the study area (C. fluminea $=$ $26.66 \mathrm{~mm} \pm 0.68 ;$ P. littoralis $=69.59 \mathrm{~mm} \pm 2.72 ; U$. delphinus $=$ $50.70 \mathrm{~mm} \pm 2.56)$.

Intact shells of recently dead organisms were collected in the study area and manually cleaned to remove any traces of soft tissues, dried at $50{ }^{\circ} \mathrm{C}$ for $48 \mathrm{~h}$, weighted, and had their length measured to nearest $0.1 \mathrm{~mm}$. Afterwards, shells were put inside net bags of $10 \mathrm{~mm}$ of mesh size ( $\mathrm{n}=10$ per treatment), and placed in a fixed location at the river bottom ( $70 \mathrm{~cm}$ deep) where they remained for two months in the summer (July and August). The summer in River Minho yields the highest rates of recruitment by many invertebrate species, with the colonization of bare substrate occurring in less than two months (see Sousa et al., 2007, 2008b; Novais et al., 2015a). At the end of the experiment, the bag contents were collected and sieved using a $500 \mu \mathrm{m}$ sieve, and the macroinvertebrates associated with the empty shells were sorted and fixed in $70 \%$ ethanol. Posteriorly, the organisms were identified to the lowest practical taxonomic level following Tachet et al. (2003) and counted. The fauna associated to each treatment was displayed in a matrix that was referred as taxa by treatment database. 


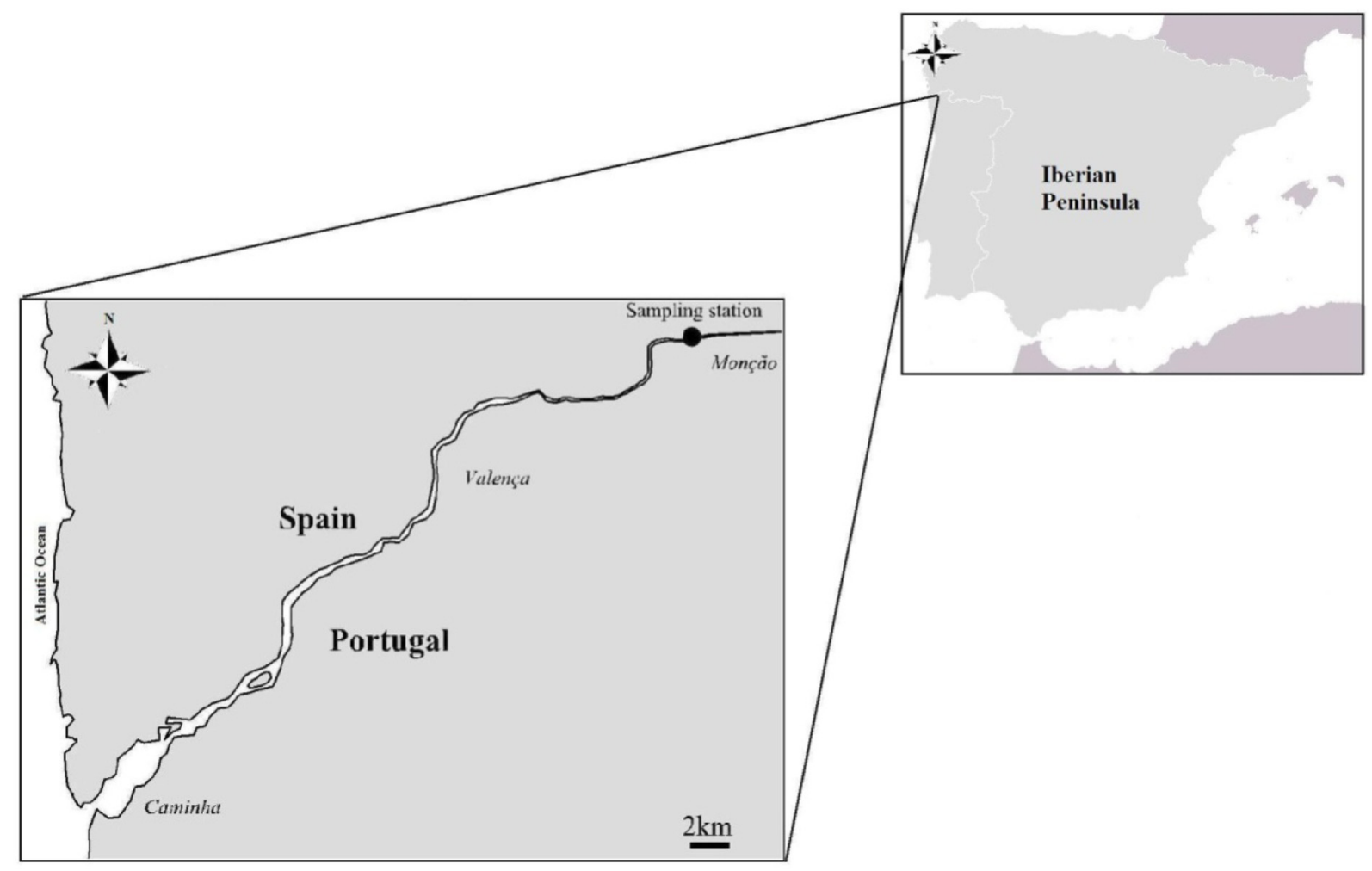

Fig. 1. Map of the River Minho (North-west Iberian Peninsula) showing the location of the experiment.

\subsection{Trait-based approach}

All the species associated to the different treatments were analyzed according to 6 traits distributed across 39 categories, selected from Tachet et al. (2003) (Table 1). The selected traits cover life history, physiological and morphological characteristics and environmental preferences. These traits were selected taking into account their importance for the structure and functioning of the benthic compartment. A standardized fuzzy coding approach (based on Tachet et al., 2003) that included scores from 0 to 5 was used to the functional characterization of the taxa associated to the different treatments. Posteriorly, these scores were standardized into a scale from 0 to 1 , in order to give the same weight to each trait. This resulted in a taxa trait database with 30 taxa and 39 trait categories.

The community-weighted mean trait (CWM) was assessed in order to determine the dominant trait categories of each invertebrate assemblage associated to the different treatments. The CWM was assessed using the taxa trait database and the taxa by treatment database that was relativized (i.e. conversion of the species absolute abundance into relative abundance) in the Excel Macro (Lepš et al., 2006; http:// botanika.bf.jcu.cz/suspa/FunctDiv.php).

Functional diversity (FD) of the associated macroinvertebrate assemblages and single traits were assessed using the Rao's quadratic entropy (FRAO) (Rao, 1982). FRAO was assessed using the taxa trait database and the relativized taxa by treatment matrix, via the Excel Macro (Lepš et al., 2006).

\subsection{Data analysis}

To evaluate the influence of the substrate on the density of the associated fauna provided by the different treatments through the taxonomic approach, a one-way PERMANOVA (type-III) was performed, with treatment (seven levels: Cor, Pot, Uni, CorPot, CorUni, UniPot, CorUniPot) as a fixed factor.
Table 1

Traits and traits categories used to classify the associated macroinvertebrates.

\begin{tabular}{|c|c|}
\hline Trait & Modalities \\
\hline Feeding & $\begin{array}{l}\text { Fine sediment }+ \text { microorganisms } \\
\text { Detritus }<1 \mathrm{~mm} \\
\text { Plant detritus }>1 \mathrm{~mm} \\
\text { Living microphytes } \\
\text { Living macrophytes } \\
\text { Dead animal }>1 \mathrm{~mm} \\
\text { Living microinvertebrates } \\
\text { Living macroinvertebrates } \\
\text { Vertebrates }\end{array}$ \\
\hline Locomotion & $\begin{array}{l}\text { Flier } \\
\text { Surface swimmer } \\
\text { Swimmer } \\
\text { Crawler } \\
\text { Burrower (epibenthic) } \\
\text { Interstitial (endobenthic) } \\
\text { Temporarily attached }\end{array}$ \\
\hline Life cycle & $\begin{array}{l}\leq 1 \text { year } \\
>1 \text { year }\end{array}$ \\
\hline Flow & $\begin{array}{l}\text { Null } \\
\text { Slow }\left(<25 \mathrm{~cm} \mathrm{~s}^{-1}\right) \\
\text { Moderate }\left(25-50 \mathrm{~cm} \mathrm{~s}^{-1}\right) \\
\text { Fast }\left(>50 \mathrm{~cm} \mathrm{~s}^{-1}\right)\end{array}$ \\
\hline Microhabitats & $\begin{array}{l}\text { Flags/boulders/cobbles/pebbles } \\
\text { Gravel } \\
\text { Sand } \\
\text { Silt } \\
\text { Macrophytes } \\
\text { Microphytes } \\
\text { Twigs/roots }\end{array}$ \\
\hline Size & $\begin{array}{l}<2.5 \mathrm{~mm} \\
2.5-5 \mathrm{~mm} \\
5-10 \mathrm{~mm} \\
10-20 \mathrm{~mm} \\
20-40 \mathrm{~mm} \\
40-80 \mathrm{~mm} \\
>80 \mathrm{~mm}\end{array}$ \\
\hline
\end{tabular}


The ecological indexes, species richness (S), Shannon-Wiener diversity index $\left(\mathrm{H}^{\prime}\right)$, Simpson diversity index and Pielou's evenness index $\left(\mathrm{J}^{\prime}\right)$ of the associated macroinvertebrate assemblages were calculated using the DIVERSE function

To evaluate the influence of the substrate on the associated fauna provided by the different treatments through the trait-based approach, the same design of the PERMANOVA previously described was used, with treatment (seven levels: Cor, Pot, Uni, CorPot, CorUni, UniPot, CorUniPot) as a fixed factor, to compare the community-weighted mean trait (CWM) categories, the functional diversity (FRAO) and the functional diversity of the single traits (i.e. feeding, locomotion, life cycle, flow, microhabitats and size).

Before each PERMANOVA analysis the variables were always normalised without data transformation and a resemble matrix based on the Euclidean distances was calculated. In all PERMANOVA tests a statistical significance of variance of $(\alpha=0.05)$ with 9999 permutations of residuals within a reduced model was considered. When the number of permutations were lower than 150 , the Monte Carlo p-value was considered. For significant results obtained by PERMANOVA, pairwise comparisons were also performed.

PRIMER software (vers. 6.1.6, PRIMER-E Ltd., Plymouth, U.K.) with PERMANOVA + 1.0.1 add-on (Anderson et al., 2008) was used for all statistical tests and analyses.

\section{Results}

\subsection{Taxonomic composition}

A total of 2961 individuals corresponding to 30 macroinvertebrate taxa of four phyla (Annelida, Arthropoda, Mollusca, Platyhelminthes) were associated with the empty bivalve shells (Table S1). Insects (15 taxa) and molluscs (10 taxa) were the most diverse groups, representing $83.3 \%$ of the taxa observed. The gastropod B. tentaculata (46.1\%), the planarians of the Tricladida order $(11.2 \%)$, the water hoglouse Asellus aquaticus (9.8\%) and the Asian clam C. fluminea (9.4\%) were the most abundant taxa (Table 2).

Of all the 30 macroinvertebrate taxa associated to the treatments, only B. tentaculata (Pseudo-F $=3.9, \mathrm{p}<0.01$ ) and Physella acuta (Pseudo- $\mathrm{F}=2.5, \mathrm{p}<0.05$ ) were significant different in the comparison between treatments (Table 2 ).

The density of the associated fauna was significantly different in the comparison between treatments. Overall, the heterogeneous treatments attracted a higher density of individuals than the homogeneous treatments (Pseudo-F $=2.52, \mathrm{p}<0.01$ ); the treatment UniPot showed the highest density values (average $\pm \mathrm{SD})\left(605.0 \pm 262.43 \mathrm{ind} \cdot \mathrm{m}^{-2}\right)$, followed by CorUni $\left(460.0 \pm 251.57\right.$ ind $\cdot \mathrm{m}^{-2}$ ) (Fig. 2). Pairwise comparisons indicated that most of the differences were related to the higher values observed for the treatment UniPot.

The highest species richness was also observed for the treatment UniPot $(8.60 \pm 2.32)$, followed by the treatment CorUniPot $(8.30 \pm$ 1.34) (Fig. 2). CorUniPot ( $1.62 \pm 0.20 ; 0.74 \pm 0.08$ ) had the highest Shannon-Wiener and Simpson diversity values, respectively, followed by the treatment Pot $(1.58 \pm 0.33 ; 0.73 \pm 0.12)$ (Fig. 2$)$. Whereas the treatment Pot had the highest evenness $(0.78 \pm 0.11)$, followed by the treatment CorUniPot $(0.77 \pm 0.09)$ (Fig. 2$)$. Nevertheless, no significant differences were observed for the ecological indexes.

\subsection{Trait composition}

The CWM showed patterns in the distribution of some trait categories associated to the different treatments. Significantly higher values were observed for the size trait categories $<2.5 \mathrm{~mm}$ (Pseudo- $\mathrm{F}=1.69$; $\mathrm{p}<0.05$ ) and $>80 \mathrm{~mm}$ (Pseudo-F $=4.64 ; \mathrm{p}<0.001$ ), the life cycle trait category $>1$ year (Pseudo-F $=3.92 ; \mathrm{p}<0.01$ ), and the flow trait category moderate (Pseudo- $F=5.57 ; \mathrm{p}<0.001$ ) for the homogeneous treatments in the comparison between treatments (Fig. 3). Pairwise

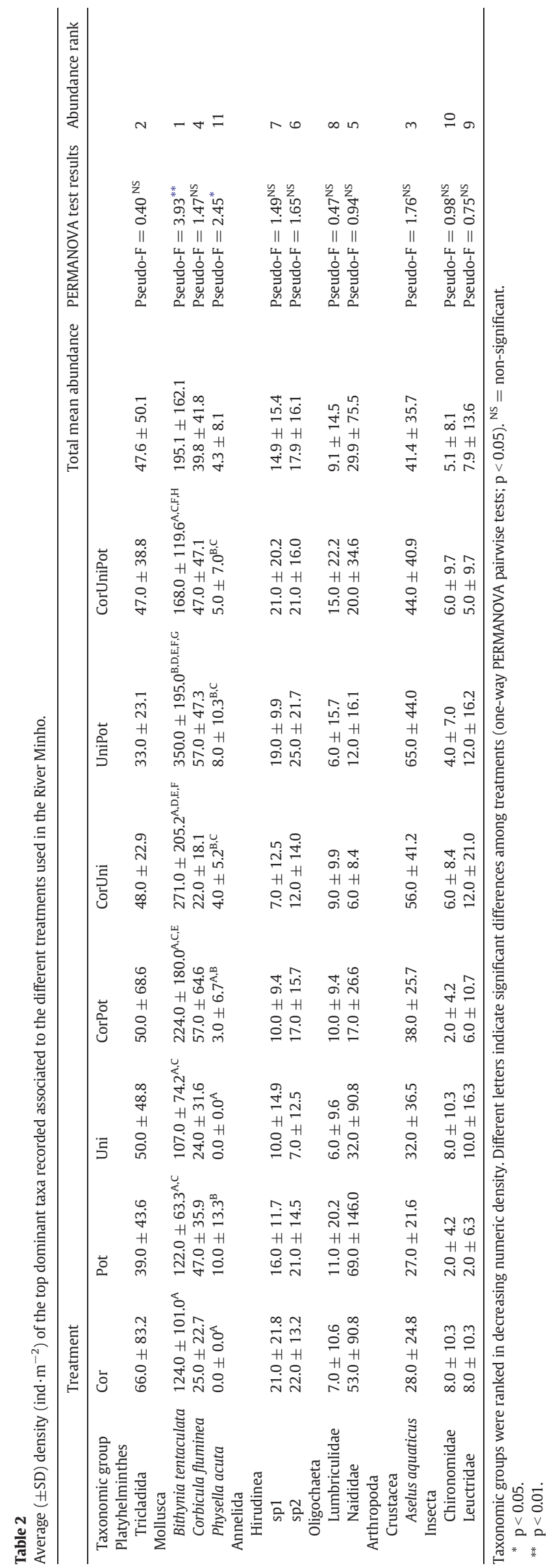



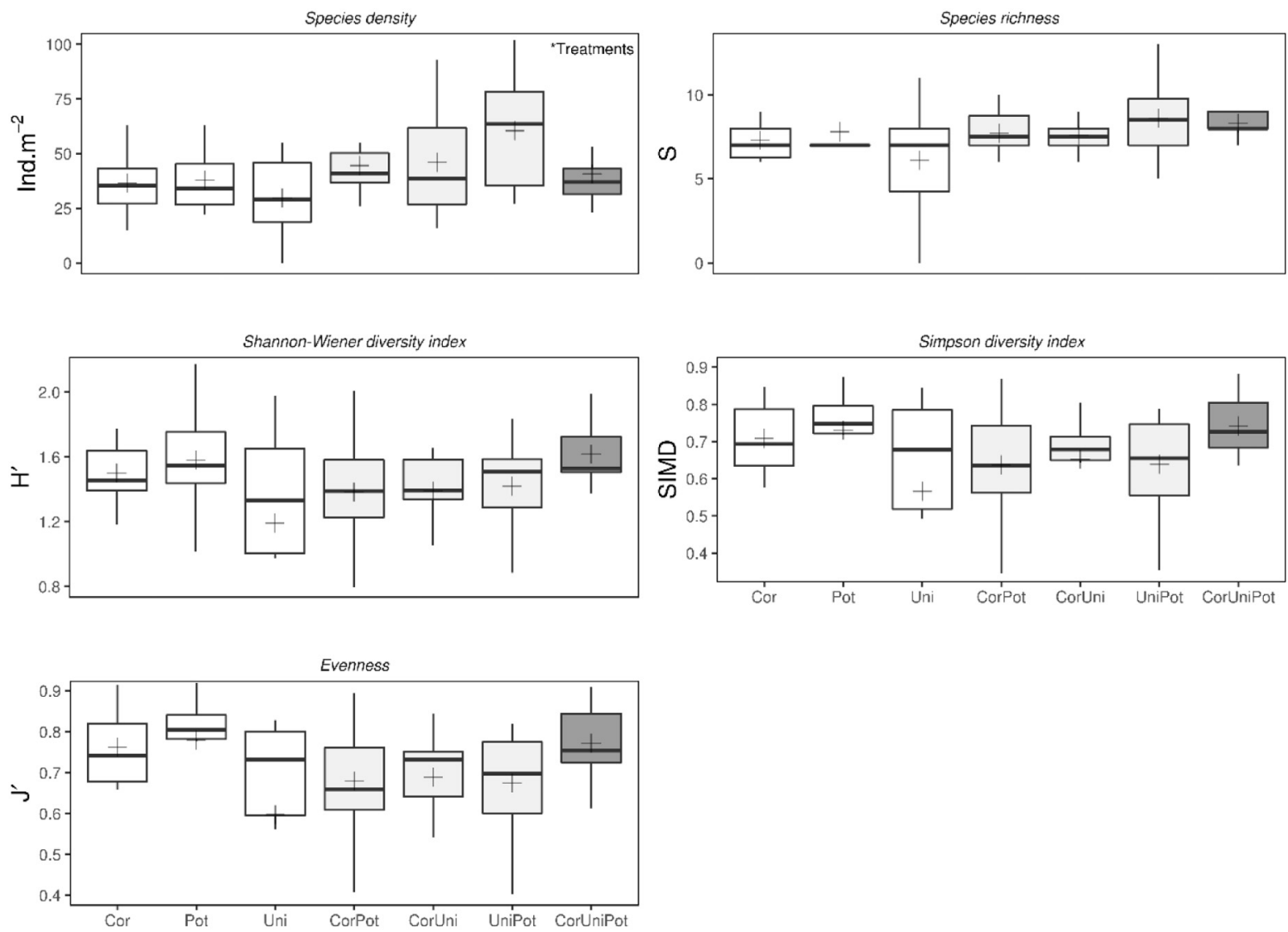

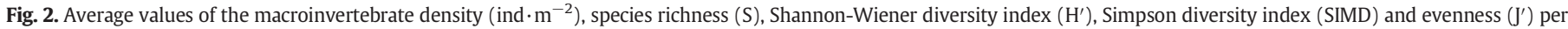
treatment. * indicates significant differences between treatments (one-way PERMANOVA; $\mathrm{p}<0.05$ ).

comparisons indicated that most of the differences observed for these categories were related to the higher values observed for the treatments Cor and Pot. Conversely, significantly higher values were observed for the life cycle trait category $<1$ year (Pseudo- $F=3.10 ; \mathrm{p}<0.01$ ), the microhabitats trait category microphytes (Pseudo-F $=4.29 ; \mathrm{p}<0.01$ ), and the flow trait categories null (Pseudo-F $=2.97 ; \mathrm{p}<0.05$ ) and slow (Pseudo-F $=2.19 ; \mathrm{p}<0.05$ ) for the heterogeneous treatments in the comparison among treatments (Fig. 4). Pairwise comparisons indicated that most of the differences were related to the higher values observed for the treatments CorUni and UniPot. No significant differences for the other trait categories in the comparison between treatments were detected.

The FRAO did not present significant differences between treatments. Overall, FRAO showed higher values for the treatment CorUniPot (0.72), followed by Pot $(0.70)$ (Table 3 ). The FD have not differed for most of the traits in the comparison between treatments (Table 3 ). The only exception was for the Flow in which the higher values were observed for the treatment Cor $(0.20)$, followed by CorUniPot (0.19) and Pot (0.19). Mostly, the treatments CorUniPot, Pot and Cor had the higher values of FD considering the analyzed traits.

\section{Discussion}

The results of the present study showed that the density of the associated macroinvertebrates was significantly higher in the heterogeneous treatments, particularly with two shell identities.

Higher 3D complexity often generates an increase in the density and diversity of the associated fauna, given that more diverse and physically complex substrata may harbor several species with different niche preferences (Stewart et al., 2003). The pattern observed for density was different of what was expected, with the highest values observed for the treatments with two shell identities instead of the treatments with three shell identities. The other metrics (i.e. richness and diversity) did not vary among treatments.

The species used as substrate in the study have different sizes, shapes, and arrangements contributing to physical structures with different heterogeneities. P. littoralis shells have a higher volume, different shape (more curved) and rougher structure, compared to U. delphinus and C. fluminea shells. U. delphinus present an intermediate shell volume and their shells are very thin and delicate, whereas $C$. fluminea have hard shells with smaller volume compared to the two aforementioned species. The spatial organization of different bivalve shells can increase the heterogeneity of the substrate; however, our results showed that habitat complexity provided by the selected empty bivalves shells contributed to attract similar, but denser macroinvertebrate assemblages.

An overall dominance of the faucet snail B. tentaculata was observed in all treatments corroborating a previous study performed in the area (Ilarri et al., 2015a); this also suggests that the species is favored by the provision of hard substrata. The faucet snail, and unlike many other snails, has two feeding modes, feeding mainly on periphyton (through grazing) and phytoplankton (through suspension feeding) (Brendelberger and Jürgens, 1993). Shells can depurate nutrients such as $\mathrm{N}$ and $\mathrm{P}$, which can stimulate periphyton growth (Ricciardi et al., 1995). Several physical and biological characteristics (e.g. accumulations of biodeposits, provision of refuge and substrate complexity) provided by the deposition of bivalve shells may have contributed to this positive interaction. Also, B. tentaculata egg deposition is frequently observed on the selected bivalve shells (authors personal observation) in the study area, suggesting that the species also uses the shells available surface for juvenile recruitment. The faucet snail had a higher density mainly in the more heterogeneous treatments. 

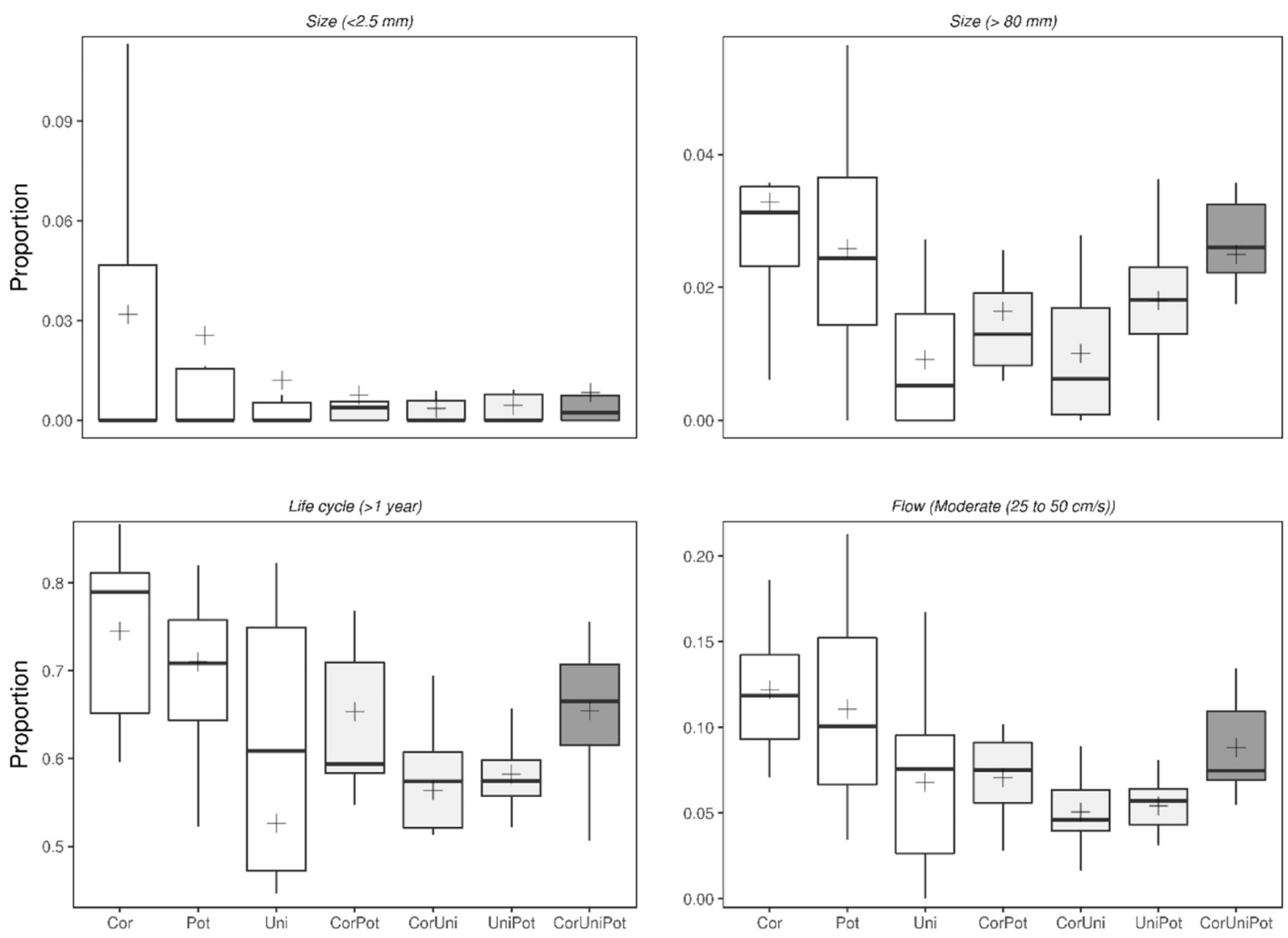

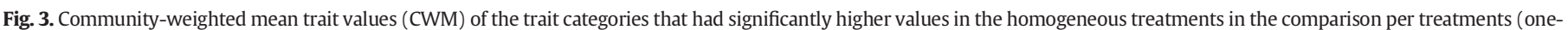
way PERMANOVA; $\mathrm{p}<0.05$ ).

The trait-based approach showed that some trait categories such as size, life cycle, microhabitats and flow responded differently to the distinct treatments. Habitat heterogeneity can contribute also, on a small spatial scale, to the species diversity and this can have implications to ecosystem functioning (Hewitt et al., 2008). It can be considered as an environmental filter in which the species have to develop suitable traits in order to persist in a specific habitat (Leung, 2015).

The CWM indicated that the smaller (size category of $<2.5 \mathrm{~mm}$ ) and largest animals (size category of $>80 \mathrm{~mm}$ ) were more abundant in homogeneous treatments. Normally, the habitat complexity can filter the size of the individuals living in it, with complex habitats supporting higher densities of small-bodied individuals (McAbendroth et al., 2005); however, in our study the highest density of smallest animals was recorded in the less complex (homogeneous) treatments. This situation may indicate that those treatments are already complex enough, and that the heterogeneous treatments did not confer any additional protection for the smallest organisms. In fact, freshwater bivalve shell structure is known to provide refuge for small macroinvertebrates (Ilarri et al., 2012; Ilarri et al., 2015a; Novais et al., 2015a), and the diversity in the composition of the shell structure (shell identity) might not be relevant for the very small organisms. However, the volume of shells might also play a significant role in attracting small organisms. Gutiérrez et al. (2003) suggest that this characteristic is relevant for shell dwellers since it may determine the space available and consequently the maximum size of the organisms. Indeed, the treatment Cor, that had the smallest shell volume, had the highest density of small individuals. It is possible that in the present study the presence of macroinvertebrates of small size categories was more closely associated with the shell volume than with the composition of the shells. Future studies should be conducted to disentangle the effects of volume and area on the associated fauna.
The higher proportion of larger invertebrates (>80 mm) in homogeneous treatments corroborates previous studies (Posey, 1987; Leung, 2015) that suggest that the maximum size decrease with the increase in complexity. The high density of both small and large invertebrates in the same area might indicates that these can be functioning as a refuge for juveniles and as breeding site for adults and this could be a possible explanation to $B$. tentaculata overall dominance in all treatments. It is also possible that the high density of small organisms attracts larger invertebrate predators thus generating a marked bimodal size distribution in those systems.

High proportion of short-lived species in a system indicates unstable and higher disturbance conditions (Van der Linden et al., 2012; Veríssimo et al., 2012), and this pattern was observed in heterogeneous treatments, suggesting that they might need more time to achieve community stability or that the conditions are less stable probably due to more complex friction force in the heterogeneous than in homogeneous substrates. In fact, this might be related also to the results observed for the flow trait categories. The null and slow flow were associated to the treatments that had a higher habitat heterogeneity (higher friction, lower flow), whereas the moderate category was associated to the homogeneous treatments (less friction, higher flow). The relationship between water flow and macroinvertebrate assemblages is straightforward, with smaller values of flow enhancing the abundance of invertebrates associated to slow flows and vice-versa (Wood and Petts, 1994; Miller and Golladay, 1996). Also, the differences in shells' hardness among species may have contributed to the observed patterns. $U$. delphinus shells are more brittle than the other two species (Ilarri et al., 2015b) and this probably lead to a faster deterioration.

We detected an increase in the proportion of microphyte-associated organisms to the increase in habitat heterogeneity corroborating previous studies (Gosselain et al., 2005). The roughness and different texture of the substrate are important features for microphytes colonization 

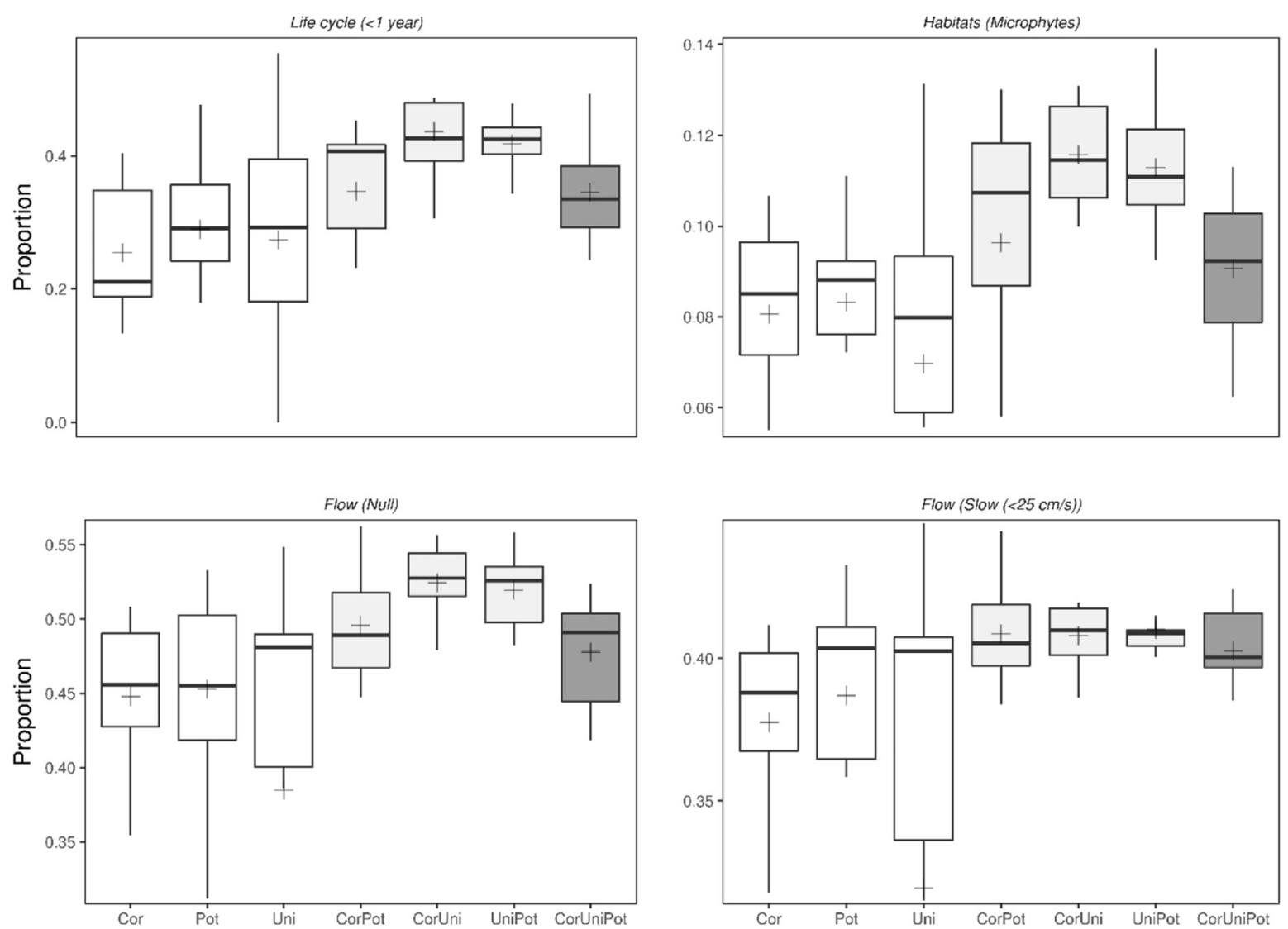

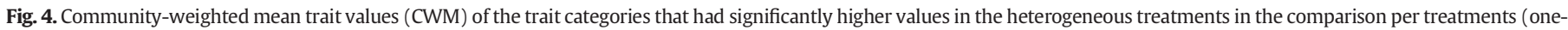
way PERMANOVA; $\mathrm{p}<0.05$ ).

(Bergey, 1999). The substrate roughness can favor the organic matter accumulation due to the presence of crevices (Johnson, 1994). In this regard, a high abundance of microphytes in the heterogeneous treatments was expected considering the higher complexity and diversity of textures provided by the presence of different shells identities.

Functional diversity measures the diversity of species traits (Van der Linden et al., 2012). It is related to the wide array of functional groups in an ecosystem, and can be associated to the higher productivity and use of resources, and better performance of the community (Petchey, 2003; Heino, 2005). On the other hand, habitat heterogeneity can lead to the increase in the amount of functional trait variability and, consequently, contribute to the variation of the functional diversity (Dimitriadis et al., 2012). In the present study, the FRAO outputs revealed similarities of the associated macroinvertebrate assemblages among treatments, with no significant differences detected, suggesting that the FRAO did not respond to the structural complexity gradient. This situation suggests that the structural heterogeneity, despite of affecting some trait categories, did not increase the functional diversity of the associated macroinvertebrate assemblages. Hence, habitat homogeneity, that has been observed in the River Minho as a gradual effect to the introduction of $C$. fluminea, will not negatively affect the macroinvertebrates colonizing empty shells, given that the structure of $C$. fluminea shells were able to maintain the functional diversity of the macroinvertebrate assemblages.

To date, few studies have evaluated the influence of the habitat heterogeneity in the functional diversity (but see Dolbeth et al., 2013; Leung, 2015; Wong and Dowd, 2015), with this study being the first to investigate this topic using empty freshwater bivalve shells. Differently from our results, most of the previous studies observed a positive relationship in the FD with increasing in habitat heterogeneity (see Dolbeth et al., 2013; Wong and Dowd, 2015). Thus, our results bring novel evidences that habitat heterogeneity effects on associated fauna

Table 3

Mean and one-way PERMANOVA test results of the Rao's quadratic entropy (FRAO) and functional trait diversity (FD) per treatment.

\begin{tabular}{|c|c|c|c|c|c|c|c|c|}
\hline Treatment & Cor & Pot & Uni & CorPot & CorUni & UniPot & CorUniPot & PERMANOVA \\
\hline FRAO & 0.68 & 0.70 & 0.54 & 0.62 & 0.63 & 0.62 & 0.72 & Pseudo- $\mathrm{F}=1.45^{\mathrm{NS}}$ \\
\hline \multicolumn{9}{|l|}{ FD } \\
\hline Trait/treatment & Cor & Pot & Uni & CorPot & CorUni & UniPot & CorUniPot & PERMANOVA \\
\hline Feeding & 0.52 & 0.50 & 0.39 & 0.43 & 0.44 & 0.41 & 0.53 & Pseudo- $\mathrm{F}=1.78^{\mathrm{NS}}$ \\
\hline Locomotion & 0.35 & 0.36 & 0.25 & 0.31 & 0.28 & 0.28 & 0.36 & Pseudo-F $=1.55^{\mathrm{NS}}$ \\
\hline Life cycle & 0.27 & 0.29 & 0.23 & 0.26 & 0.26 & 0.26 & 0.31 & Pseudo-F $=1.05^{\mathrm{NS}}$ \\
\hline Flow & $0.20^{\mathrm{a}}$ & $0.19^{\mathrm{ac}}$ & $0.14^{\text {ace }}$ & $0.16^{\text {acef }}$ & $0.14^{\text {bdefg }}$ & $0.14^{\text {becfgi }}$ & $0.19^{\text {acefhj }}$ & Pseudo-F $=2.25^{*}$ \\
\hline Microhabitats & 0.32 & 0.35 & 0.25 & 0.31 & 0.29 & 0.30 & 0.36 & Pseudo-F $=1.97^{\mathrm{NS}}$ \\
\hline Size & 0.51 & 0.54 & 0.38 & 0.45 & 0.45 & 0.48 & 0.54 & Pseudo-F $=2.04^{\mathrm{NS}}$ \\
\hline
\end{tabular}

Different letters indicate significant differences among treatments (one-way PERMANOVA pairwise tests; $\mathrm{p}<0.05$ ). ${ }^{\mathrm{NS}}=$ non-significant.

* $\mathrm{p}<0.05$. 
is not straightforward, being highly context dependent and more complex than previously thought.

\section{Conclusion}

Homogenization can lead to changes on the physical structure of habitats. By studying the changes in the substrate heterogeneity (through different bivalve shells identities) on the structure of macroinvertebrate assemblages it was possible to conclude that the habitat heterogeneity positively affects the density of macroinvertebrates, favoring the organisms that feed on microphytes, inhabit areas with null to slow water flow and that have short live cycles. Also, within a habitat homogenization scenario, that included the effects of a dominant non-native species, it was also possible to infer that the structural complexity provided by the empty shells of the Asian clam $C$. fluminea was able to maintain the associated macroinvertebrate assemblages functionally diverse.

Supplementary data to this article can be found online at https://doi. org/10.1016/j.scitotenv.2018.04.070.

\section{Acknowledgments}

This study was conducted as part of the project FRESCHO: Multiple implications of invasive species on Freshwater Mussel coextinction processes, supported by FCT (contract: PTDC/AGRFOR/1627/2014). M Ilarri is supported by a Post-doc grant (SFRH/BPD/90088/2012) from the Portuguese Foundation for Science and Technology - FCT through POPH/ FSE funds.

\section{References}

Anderson, M.J., Gorley, R.N., Clarke, K.R., 2008. PERMANOVA + for PRIMER: Guide to Software and Statistical Methods. PRIMER-E, Plymouth.

Araujo, R., Moreno, D., Ramos, M., 1993. The Asiatic clam Corbicula fluminea (Müller 1774) (Bivalvia: Corbiculidae) in Europe. Am. Malacol. Bull. 10, 39-49.

Bergey, E.A., 1999. Crevices as refugia for stream diatoms: effects of crevices size on abraded substrates. Limnol. Oceanogr. 44, 1522-1529.

Bódis, E., Tóth, B., Szekeres, J., Borza, P., Sousa, R., 2014. Empty native and invasive bivalve shells as benthic habitat modifiers in a large river. Limnologica 49, 1-9.

Brendelberger, H., Jürgens, S., 1993. Suspension feeding in Bithynia tentaculata (Prosobanchia, Bithyniidae), as affected by body size, food and temperature. Oecologia 94 (1), 36-42.

Burdon, D., Callaway, R., Elliott, M., Smith, T., Wither, A., 2014. Mass mortalities in bivalve populations: a review of the edible cockle Cerastoderma edule (L.). Estuar. Coast. Shelf Sci. 150, 217-280.

Clare, D.S., Robinson, L.A., Frid, C.L.. 2015. Community variability and ecological functioning: 40 years of change in the North Sea benthos. Mar. Environ. Res. 107, 24-34.

Costa-Dias, S., Freitas, V., Sousa, R., Antunes, C., 2010. Factors influencing epibenthic assemblages in the Minho estuary (NW Iberian Peninsula). Mar. Pollut. Bull. 61, 240-246.

Dimitriadis, C., Evagelopoulos, A., Koutsoubas, D., 2012. Functional diversity and redundancy of soft bottom communities in brackish waters areas: local vs regional effects. J. Exp. Mar. Biol. Ecol. 426, 53-59.

Dolbeth, M., Cardoso, P., Grilo, T., Raffaelli, D., Pardal, M.A., 2013. Drivers of estuarine benthic species distribution patterns following a restoration of a seagrass bed: a functional trait analyses. Mar. Pollut. Bull. 72, 47-54.

Erwin, D., 2008. Macroevolution of ecosystem engineering, niche construction and diversity. Trends Ecol. Evol. 23, 304-310.

Gosselain, V., Hudon, C., Cattaneo, A., Gagnon, P., Planas, D., Rochefort, D., 2005. Physical variables driving epiphytic algal biomass in a dense macrophyte bed of the St. Lawrence River (Quebec, Canada). Hydrobiologia 534, 11-22.

Gutiérrez, J., Jones, C., Strayer, D., Iribarne, O., 2003. Mollusks as ecosystem engineers: the role of shell production in aquatic habitats. Oikos 101, 79-90.

Haag, W.R., Warren Jr., M.L., 2008. Effects of severe drought on freshwater mussel assemblages. Trans. Am. Fish. Soc. 137, 1165-1178

Heino, J., 2005. Functional diversity of macroinvertebrate assemblages along major ecological gradients of boreal headwater streams. Freshw. Biol. 50, 1578-1587.

Hewitt, J.E., Thrush, S.F., Dayton, P.D., 2008. Habitat variation, species diversity and ecological functioning in a marine system. J. Exp. Mar. Biol. Ecol. 366, 116-122.

Ilarri, M., Antunes, C., Guilhermino, L., Sousa, R., 2011. Massive mortality of the Asian clam Corbicula fluminea in a highly invaded area. Biol. Invasions 13, 277-280.

Ilarri, M., Freitas, F., Costa-Dias, S., Antunes, C., Guilhermino, L., Sousa, R., 2012. Associated macrozoobenthos with the invasive Asian clam Corbicula fluminea. J. Sea Res. 72 $113-120$.

Ilarri, M.I., Souza, A.T., Antunes, C., Guilhermino, L., Sousa, R., 2014. Influence of the Asian clam Corbicula fluminea (Bivalvia: Corbiculidae) on estuarine epibenthic assemblages. Estuar. Coast. Shelf Sci. 143,12-19.
Ilarri, M., Souza, A., Modesto, V., Guilhermino, L., Sousa, R., 2015a. Differences in the macrozoobenthic fauna colonising empty bivalve shells before and after invasion by Corbicula fluminea. Mar. Freshw. Res. 66, 549

Ilarri, M., Souza, A., Sousa, R., 2015b. Contrasting decay rates of freshwater bivalves' shells: aquatic versus terrestrial habitats. Limnologica 51, 8-14.

Johnson, L.E., 1994. Enhanced settlement on microtopographical high points by the intertidal red alga Halosaccion glandiforme. Limnol. Oceanogr. 39 (8), 1893-1902.

Lepš, J., De Bello, F., Lavorel, L., Berman, S., 2006. Quantifying and interpreting functional diversity of natural communities: practical considerations matter. Preslia 78, 481-501.

Leung, J.Y.S., 2015. Habitat heterogeneity affects ecological functions of microbenthic communities in a mangrove: implication for the impact of restoration and afforestation. Glob. Ecol. Conserv. 4, 423-433.

Leuven, R.S.E.W., Collas, F.P.L., Koopman, K.R., Matthews, J., Van der Velde, G., 2014. Mass mortality of invasive zebra and quagga mussels by desiccation during severe winter conditions. Aquat. Invasions 9 (3), 243-252.

Linares, M.S., Callisto, M., Marques, J.C., 2017. Invasive bivalves increase benthic communities complexity in neotropical reservoirs. Ecol. Indic. 75, 279-285.

McAbendroth, L., Ramsay, P.M., Foggo, A., Rundle, S.D., Bilton, D.T., 2005. Does macrophytes fractal complexity drive invertebrate diversity, biomass, and body size distributions? Oikos 111 (2), 279-290.

Miller, A.M., Golladay, S.W., 1996. Effects of spates and drying on macroinvertebrate assemblages of an intermittent and a perennial prairie stream. J. N. Am. Benthol. Soc. $15,670-689$

Novais, A., Souza, A., Ilarri, M., Pascoal, C., Sousa, R., 2015a. Facilitation in the low intertidal: effects of an invasive species on the structure of an estuarine macrozoobenthic assemblage. Mar. Ecol. Prog. Ser. 522, 157-167.

Novais, A., Souza, A., Ilarri, M., Pascoal, C., Sousa, R., 2015b. From water to land: how an invasive clam may function as a resource pulse to terrestrial invertebrates. Sci. Total Environ. 538, 664-671.

Novais, A., Pascoal, C., Sousa, R., 2017. Effects of invasive aquatic carrion on soil chemistry and terrestrial microbial communities. Biol. Invasions 19, 2491-2502.

Petchey, O.L., 2003. Integrating methods that investigate how complementarity influences ecosystem functioning. Oikos 101, 323-330.

Posey, M.H., 1987. Influence of relative mobilities on the composition of benthic communities. Mar. Ecol. Prog. Ser. 39, 99-104.

Rao, C.R., 1982. Diversity and dissimilarity coefficients: a unified approach. Theor. Popul. Biol. 21, 24-43.

Ricciardi, A., Whoriskey, F.G., Rasmussen, J.B., 1995. Predicting the intensity and impact of Dreissena infestation on native unionid bivalves from Dreissena field density. Can. J. Fish. Aquat. Sci. 52, 1449-1461.

Sousa, R., Guilhermino, L., Antunes, C., 2005. Molluscan fauna in the freshwater tidal area of the River Minho estuary, NW of Iberian Peninsula. Ann. Limnol. Int. J. Limnol. 41, 141-147.

Sousa, R., Antunes, C., Guilhermino, L., 2007. Species composition and monthly variation of the Molluscan fauna in the freshwater subtidal area of the River Minho estuary. Estuar Coast Shelf Sci. 75, 90-100.

Sousa, R., Dias, S., Freitas, V., Antunes, C., 2008a. Subtidal macrozoobenthic assemblages along the River Minho estuarine gradient (north-west Iberian Peninsula). Aquat. Conserv. 18, 1063-1077.

Sousa, R., Nogueira, A., Gaspar, M., Antunes, C., Guilhermino, L., 2008b. Growth and extremely high production of the non-indigenous invasive species Corbicula fluminea (Müller, 1774): possible implications for ecosystem functioning. Estuar. Coast. Shelf Sci. 80, 289-295.

Sousa, R., Dias, S., Guilhermino, L., Antunes, C., 2008c. Minho River tidal freshwater wetlands: threats to faunal biodiversity. Aquat. Biol. 3, 237-250.

Sousa, R., Gutiérrez, J.L., Aldridge, D.C., 2009. Non-indigenous invasive bivalves as ecosystem engineers. Biol. Invasions 11, 2367-2385.

Sousa, R., Varandas, S., Cortes, R., Teixeira, A., Lopes-lima, M., Machado, J., Guilhermino, L., 2012. Massive die-offs of freshwater bivalves as resources pulses. Ann. Limnol. Int. J. Limnol. 48, 105-112.

Stewart, T.W., Shumaker, T.L., Radzio, A., 2003. Linear and nonlinear effects of habitat structure on composition and abundances in the macroinvertebrate community of a large river. Am. Midl. Nat. 149, 293-305.

Tachet, H., Richoux, P., Bournaud, M., Usseglio-Polaterra, P., 2003. Invertébrés D'Eau Douce: Systématique, Biologie, Écologie. CNRS Editions, Paris.

Van der Linden, P., Patrício, J., Marchini, A., Cid, N., Neto, J.M., Marques, J.C., 2012. A biological trait approach to assess the functional composition of subtidal benthic communities in an estuarine ecosystem. Ecol. Indic. 20, 121-133.

Van der Linden, P., Marchini, A., Dolbeth, M., Patrício, J., Veríssimo, H., Marques, J.C., 2016. The performance of trait-based indices in an estuarine environment. Ecol. Indic. 61 (2), 378-389.

Veríssimo, H., Bremner, J., Garcia, C., Patrício, J., Van der Linden, P., Marques, J.C., 2012. Assessment of the subtidal macrobenthic community functioning of a temperate estuary following environmental restoration. Ecol. Indic. 23, 312-322.

Weigel, B., Blenckner, T., Bonsdorff, E., 2016. Maintained functional diversity in benthic communities in spite of diverging functional identities. Oikos 125 (10), 1421-1433.

Wong, M.C. Dowd, M. 2015. Patterns in taxonomic and functional diversity of macrobenthic invertebrates across seagrass habitats: a case study in Atlantic Canada. Estuar. Coasts 38, 2323-2336.

Wood, P.J., Petts, G.E., 1994. Low flows and recovery of macroinvertebrates in a small regulated chalk stream. Regul. Rivers 9, 303-316.

Zaiko, A., Daunys, D., Olenin, S., 2009. Habitat engineering by the invasive zebra mussel Dreissena polymorpha (Pallas) in a boreal coastal lagoon: impact on biodiversity. Hegol. Mar. Res. 63, 85-94. 